BSG 2014 abstracts

\begin{tabular}{|c|c|c|c|}
\hline \multicolumn{4}{|l|}{ Symptoms } \\
\hline (7 patients) & Mean pre-treatment score (range) & Mean score in final two weeks (range) & Percent change \\
\hline Composite & $34.7(18.3-62.4)$ & $27.49(14.7-59.3)$ & $-21 \%$ \\
\hline Nausea & $2.8(0.6-4.7)$ & $2.2(0.5-4.3)$ & $-21 \%$ \\
\hline Vomiting & $1.2(0-5)$ & $1.2(0.1-5)$ & $0 \%$ \\
\hline Early satiety & $3.3(2.1-5)$ & $2.6(1.7-5)$ & $-21 \%$ \\
\hline Bloating & $2.4(1.1-3.5)$ & $1.7(0.4-3.2)$ & $-29 \%$ \\
\hline
\end{tabular}

Introduction Vagal dysfunction has been implicated in gastroparesis. Gut vagal afferents convey symptoms of nausea, bloating and early satiety but the nerve also has an antinociceptive function. GammaCore (electroCore, LLC: New Jersey) is a CE marked hand-held vagus nerve stimulator designed to selectively stimulate afferent vagal A-fibres. It is possible that in gastroparesis, stimulation of the vagus nerve as it traverses the neck might influence symptoms.

Methods Fifteen patients with severe gastroparesis awaiting Enterra (Medtronic, Minnesota) implantation agreed to a short proof of concept assessment of nVNS. Each patient was supplied with a GammaCore device programmed to deliver 150 doses, each dose lasting 90 seconds. The electrodes on the GammaCore device were positioned in line with the right cervical vagus nerve and stimulation applied three times daily. The gastroparesis multi-symptom questionnaire, that includes the symptoms of nausea, vomiting, early satiety and bloating, was completed daily for the week prior to starting treatment and daily throughout the treatment period. Symptoms were scored on a Likert scale $(1=$ none and $5=$ severe $)$. Composite and individual symptom scores were summated for the week preceding treatment and the final two weeks of the treatment period.

Results Only seven of the 15 patients complied with the treatment regimen. In six patients, the diary score cards were incomplete and two patients did not use the GammaCore.

Conclusion In this group of severely ill patients awaiting Enterra implantation, half complied with the treatment regimen. Compliant patients scored improvement in nausea, early satiety and bloating, as well as the composite gastroparesis score. This short term proof of concept assessment suggests that nVNS influences symptoms conveyed by vagal afferents. It is possible that stimulation of both left and right vagus nerves, increased stimulation amplitude, and a longer period of treatment might improve responsiveness. Future clinical trials are warranted to elucidate safety, efficacy, dose response and compliance.

Disclosure of Interest None Declared.

\section{PWE-175 COST-EFFECTIVENESS OF LINACLOTIDE (LIN) COMPARED TO ANTIDEPRESSANTS (ATDS) IN THE TREATMENT OF IRRITABLE BOWEL SYNDROME WITH CONSTIPATION (IBS-C) IN SCOTLAND}

${ }^{1} \mathrm{M}$ Fisher, ${ }^{2} \mathrm{~A}$ Walker, ${ }^{3} \mathrm{M}$ Falqués, ${ }^{4} \mathrm{M}$ Rance*, ${ }^{5} \mathrm{D}$ Taylor, ${ }^{3} \mathrm{~L}$ Lindner. ${ }^{1} W G$ Consulting, High Wycombe, UK; ${ }^{2}$ Glasgow University, Glasgow, UK; ${ }^{3}$ Almirall S. A., Barcelona, Spain; ${ }^{4}$ Almirall UK, Uxbridge, UK; ${ }^{5}$ Ironwood Pharmaceuticals, Cambridge, Massachusetts, USA

\subsection{6/gutjnl-2014-307263.435}

Introduction IBS-C is a chronic functional gastrointestinal disorder that has been shown to decrease quality of life (QoL) and work productivity, and increase activity impairment. LIN is indicated by the European Medicines Agency as the only treatment for IBS-C (covering all key symptoms) in adults and has received a positive recommendation from the Scottish Medicines
Consortium. This study sought to determine the cost-effectiveness of LIN compared with ATDs for the treatment of adults with moderate to severe IBS-C who have previously received antispasmodics and/or laxatives.

Methods A Markov model was created to estimate costs and quality-adjusted life years (QALYs) on 4-week cycles over a 5-year time horizon from the perspective of NHS Scotland. The comparator used was ATDs, reflecting Scottish current practice. Health states were based on treatment satisfaction (satisfied, moderately satisfied, not satisfied) and death. Transitions between states were based on satisfaction data from the LIN pivotal studies and the Scottish general all-cause mortality statistics. Treatment costs were calculated from the British National Formulary using the least-expensive ATD cost (amitriptyline hydrochloride $20 \mathrm{mg}$ ). NHS resource use for each state was collected from Scottish clinician interviews and combined with NHS Reference costs to obtain disease-related costs. QoL was based on the EQ-5D collected from the pivotal studies. Uncertainty was explored through extensive sensitivity analyses.

Results The base case comparing LIN with ATDs over 5 years provided an incremental cost-effectiveness ratio (ICER) value (cost per QALY) of $£ 7,370$. Scenario analyses conducted to address structural uncertainty resulted in ICERs ranging from $£ 1,204$ to $£ 10,899$. Probabilistic sensitivity analysis was also conducted using 10,000 iterations to examine parameter uncertainty over the entire model. This analysis showed that there was an $83 \%$ probability that LIN was cost-effective at a willingness to pay an ICER of $£ 20,000$ when compared with ATDs. The mean probabilistic ICER was $£ 4,606$ (mean incremental costs $£ 394$ and mean incremental QALYs 0.085).

Conclusion LIN is a cost-effective treatment for adults with moderate to severe IBS-C who have previously received antispasmodics and/or laxatives. The ICER in Scotland was calculated as $£ 7,370$ when compared with ATDs and was based on conservative assumptions. Results were robust to extensive sensitivity analyses.

Study funded by Almirall UK.

Disclosure of Interest M. Fisher Conflict with: former employee of WG Consulting, A. Walker Consultant for: WG Consulting, Conflict with: WG Consulting (Advisory Board meeting), M. Falqués Employee of: Almirall S. A., M. Rance Employee of: Almirall UK, D. Taylor Employee of: Ironwood Pharmaceuticals, L. Lindner Employee of: Almirall S. A.

\section{PWE-176 PREVALENCE, MANAGEMENT, AND HEALTHCARE BURDEN OF IRRITABLE BOWEL SYNDROME (IBS) IN SCOTLAND}

${ }^{1} \mathrm{~S}$ McTaggart, ${ }^{1} \mathrm{G}$ Wyper, ${ }^{1} \mathrm{~L}$ Harkins, ${ }^{1} \mathrm{I}$ Bishop, ${ }^{1} \mathrm{M}$ Bennie ${ }^{1},{ }^{2} \mathrm{M}$ Rance*. ${ }^{1} \mathrm{NHS}$ National Services UK, Edinburgh, UK; ${ }^{2}$ Almirall, Uxbridge, UK

\subsection{6/gutjnl-2014-307263.436}

Introduction Using real-world healthcare, routinely collected information we investigated the prevalence of irritable bowel 
syndrome (IBS) and associated consumption of healthcare resources in the Scottish National Health Service.

Methods A consultation records database covering 56 general practitioner (GP) practices in Scotland (approximately 255,000 people; $5.8 \%$ of the population) was used to identify consultations primarily due to IBS with GP or practice nurses between April 2009 and March 2011 (Read codes: IBS, constipation, diarrhoea). Read codes suggesting other causes of diarrhoea or constipation and patients with inflammatory bowel disease, diverticular disease, coeliac disease or bowel cancer were excluded. A national primary care prescription database was used to identify use of antispasmodics, laxatives or antidiarrhoeals. Referrals to outpatient clinics and acute hospital admissions between January and December 2011 in antispasmodictreated patients were also analysed using national datasets.

Results Based on consultation records, an estimated 341,180 adults ( $\geq 18$ years) in Scotland suffer from IBS, representing an estimated prevalence of $7.7 \%$ (females: 9.8\%; males: $5.5 \%$ ). Patients consulted 1.05 times during 2010/11 for IBS symptoms and consulted more frequently overall than the general population (9.3 vs. 4.7 times per annum).

During 2011, 142,738 adults received $\geq 1$ prescription for antispasmodics, most commonly mebeverine (40.1\%), hysocine butylbromide $(35.7 \%)$ or peppermint $(18.0 \%)$, giving an estimated prevalence of antispasmodic-treated IBS of 3.4\% (females: 4.7\% males: $2.0 \%$ ). One-third of these patients were also prescribed laxatives (24.5\%), the antidiarrhoeal loperamide (6.7\%) or both $(2.5 \%)$. Of the antispasmodic-treated patients, 11,645 $(9.0 \%)$ visited a gastroenterology outpatient clinic in 2011 $(11.7 \%$ of all gastroenterology clinic attendances) and 1,869 (1.3\%) were acutely admitted to hospital due to IBS or symptoms likely to be associated with IBS, most frequently constipation (80.3\%). The average length of hospital stay was 2.1 days (2.4 days for admissions due to constipation). A further 1,141 people with no record of antispasmodic prescriptions were admitted with a primary diagnosis of IBS.

Conclusion Based on consultation records, the estimated prevalence of IBS in Scotland is 7.7\%, and 3.4\% based on prescription records; these estimates exclude patients who do not consult a GP or who use laxatives alone or over-the-counter medicines. The prevalence estimates and levels of treatment in this study were consistent with other published information, and show that patients with IBS consume significant healthcare resources.

Study commissioned by Almirall UK.

Disclosure of Interest S. McTaggart Grant/research support from: This study was undertaken as a commercially contracted piece of work for Almirall UK, G. Wyper Grant/research support from: This study was undertaken as a commercially contracted piece of work for Almirall UK, L. Harkins Grant/research support from: This study was undertaken as a commercially contracted piece of work for Almirall UK, I. Bishop Grant/research support from: This study was undertaken as a commercially contracted piece of work for Almirall UK, M. Bennie Grant/research support from: This study was undertaken as a commercially contracted piece of work for Almirall UK, M. Rance Employee of: Almirall.

\section{PWE-177 A SYSTEMATIC REVIEW OF ANTIDEPRESSANTS IN IRRITABLE BOWEL SYNDROME: A QUALITATIVE ANALYSIS}

${ }^{1} \mathrm{M}$ Rance ${ }^{*},{ }^{2} \mathrm{~L}$ Lindner, ${ }^{3} \mathrm{AC}$ Ford. ${ }^{1}$ Almirall UK, Uxbridge, UK, ${ }^{2}$ Almirall S. A., Barcelona, Spain; ' Leeds University and Leeds Teaching Hospitals Trust, Leeds, UK

10.1136/gutjnl-2014-307263.437
Introduction Antidepressants have a mild analgesic effect and are commonly used in the management of irritable bowel syndrome (IBS); however, the quality of the clinical evidence to support the use of tricyclic antidepressants (TCAs) and selective serotonin inhibitors (SSRIs) in IBS is unclear. The aim of this literature review was to systematically identify and analyse published evidence on the efficacy of TCAs and SSRIs for IBS.

Methods A systematic search of the medical literature was conducted using the PubMed, Embase, and Cochrane databases. Search terms included 'irritable bowel syndrome', 'spastic colon', 'irritable colon', 'functional diseases, colon' and a mixture of agent terms - including antidepressants, tricyclic, and SSRIs. Randomised placebo-controlled trials evaluating the efficacy of antidepressants (SSRIs and TCAs) in adult patients with IBS were eligible for inclusion. Exclusion criteria included absence of placebo arm, patients $<18$ years of age, and dual publication. Only antidepressants available in the UK were included in this analysis.

Results A total of 628 unique titles and abstracts were retrieved; 581 records were excluded upon title or abstract review and 33 upon full-text review. The final review included 14 studies: 7 reporting on TCAs, 6 on SSRIs, and 1 comparing both an SSRI and a TCA vs. placebo. Agents included in the analysis were citalopram, paroxetine, fluoxetine, imipramine, amitriptyline, trimipramine and doxepin. Overall, 797 patients were included in the 14 studies, with 240 receiving a TCA, 171 receiving an SSRI and 386 receiving placebo. Of the nine studies (475 patients) that reported IBS subtype, $50 \%$ of patients had diarrhoea-predominant IBS, $32 \%$ had constipation-predominant IBS (IBS-C) and 19\% had alternating-type IBS. Treatment duration ranged from 4-12 weeks. The most common IBS-related outcome measures were for global symptom relief and improvements in abdominal pain/discomfort. Three studies reported on quality of life, while only limited data were available for treatment satisfaction. Most outcomes did not align well with those now required for FDA and EMA regulatory approval of medicines to treat IBS-C.

Conclusion The evidence base for the use of antidepressants in IBS is of low quality, making estimates of effect very uncertain. Data for the efficacy of antidepressants in IBS subtypes are especially limited. Further studies are required to support the offlabel use of antidepressants in IBS.

Study funded by Almirall UK.

Disclosure of Interest M. Rance Employee of: Almirall UK, L. Lindner Employee of: Almirall S. A., A. C. Ford: None Declared.

\section{PWE-178 IMPACT OF CHRONIC CONSTIPATION ON HEALTHCARE RESOURCE USE IN THE UK: AN ANALYSIS BASED ON ELECTRONIC MEDICAL RECORDS}

${ }^{1} \mathrm{M}$ Radford*, ${ }^{1} \mathrm{E}$ Bloomfield, ${ }^{2} \mathrm{~A}$ Joseph. 'IMS Health, London, UK; ${ }^{2}$ Shire, Eysins, Switzerland

\subsection{6/gutjnl-2014-307263.438}

Introduction Chronic constipation is a common condition with a substantial impact on health-related quality of life. The aim of this study was to assess healthcare resource use and prescription laxative costs associated with chronic constipation in the UK, using electronic medical records.

Methods The IMS Disease Analyzer database contains electronic medical records from primary healthcare practices in England and Wales. Patients were identified as having chronic constipation if they had: $\geq 2$ international classification of diseases-revision 10 (ICD-10) codes for constipation within 12 months; or 1 primary constipation diagnosis and $\geq 2$ laxative prescriptions in the 6 months before or 12 months after the constipation 